\title{
Establishing a Regional, Multisite Database for Quality Improvement and Service Planning in Community-Based Palliative Care and Hospice
}

\author{
Janet Bull, M.D., S. Yousuf Zafar, M.D., M.H.S., ${ }^{2,3}$ Jane L. Wheeler, M.S.P.H., ${ }^{2}$ \\ Matthew Harker, M.B.A., M.P.H., ${ }^{2}$ Agbessi Gblokpor, ${ }^{2}$ Laura Hanson, M.D., ${ }^{4}$ \\ Deirdre Hulihan, M.S.N., A.N.P., Rikki Nugent, M.S.N., F.N.P., \\ John Morris, M.D., and Amy P. Abernethy, M.D., ${ }^{2,3}$
}

\begin{abstract}
Background: Outpatient palliative care, an evolving delivery model, seeks to improve continuity of care across settings and to increase access to services in hospice and palliative medicine (HPM). It can provide a critical bridge between inpatient palliative care and hospice, filling the gap in community-based supportive care for patients with advanced life-limiting illness. Low capacities for data collection and quantitative research in HPM have impeded assessment of the impact of outpatient palliative care.

Approach: In North Carolina, a regional database for community-based palliative care has been created through a unique partnership between a HPM organization and academic medical center. This database flexibly uses information technology to collect patient data, entered at the point of care (e.g., home, inpatient hospice, assisted living facility, nursing home). HPM physicians and nurse practitioners collect data; data are transferred to an academic site that assists with analyses and data management. Reports to community-based sites, based on data they provide, create a better understanding of local care quality.

Current status: The data system was developed and implemented over a 2-year period, starting with one community-based HPM site and expanding to four. Data collection methods were collaboratively created and refined. The database continues to grow. Analyses presented herein examine data from one site and encompass 2572 visits from 970 new patients, characterizing the population, symptom profiles, and change in symptoms after intervention.

Conclusion: A collaborative regional approach to HPM data can support evaluation and improvement of palliative care quality at the local, aggregated, and statewide levels.
\end{abstract}

\section{Introduction}

$\mathrm{O}$ UTPATIENT PALliative CARE PRograms have been described as a "new frontier of palliative care," a mechanism for addressing a persistent and problematic gap in palliative care: lack of available nonhospice services in the community. ${ }^{1}$ These programs can deliver services in home, clinic, or long-term care settings, in a brief consultation or concurrent care model. The outpatient palliative care clinic model can play a key role in assuring continuity of care across settings; its function is particularly important at transitions when patients leave the hospital and return to their homes and communities where expert palliative care is lacking. Outpatient palliative care is becoming a bridge to hospice.

While evaluation of services provided by hospital-based palliative care programs can utilize routinely collected data from hospital databases, the assessment of services in outpatient palliative care clinics proves more difficult. Meanwhile, hospice administrative data are not necessarily applicable to the palliative care setting nor might they provide

\footnotetext{
${ }^{1}$ Four Seasons, Flat Rock, North Carolina.

${ }^{2}$ Division of Medical Oncology, Department of Medicine, ${ }^{3}$ Duke Comprehensive Cancer Center, Duke University Medical Center, Durham, North Carolina.

${ }^{4}$ Division of Geriatrics, Palliative Care Program, Department of Medicine, University of North Carolina-Chapel Hill, Chapel Hill, North Carolina.

Accepted March 31, 2010.
} 
insight for improving clinical practice and the patient experience. Outpatient palliative care is a data gap in the middle.

Historically, hospice and palliative medicine (HPM) programs have not been oriented toward research nor invested heavily in infrastructure for data management. ${ }^{2}$ Their community focus decreases interaction with traditional quality and research activities, reducing the chance that high-quality data are routinely used to inform best practice. The limited data related to the outpatient hospice experience have demonstrated improved outcomes when quality improvement programs are implemented. ${ }^{3}$

The importance of data for understanding distributions of health care practice, delivery, and spending is powerfully demonstrated by the Dartmouth Atlas, begun in 1996. These data have been used to support the fiscal case for inpatient palliative care services, as a way to better manage high-need, high-cost, high-utilization patients. ${ }^{4}$ We describe a project in North Carolina that uses a newly instituted, multisite, technology-based data collection system to: (1) examine patterns of access and utilization, symptoms, and advance care planning and (2) report on quality. This system has been developed and piloted in three community-based HPM programs and one hospital-based palliative care program, representing diverse care models.

Key questions prompting the system's development, as well as the sites' participation, were: Can we use a technologybased data collection system in these settings? What are the characteristics and symptom profiles of patients being served by community-based HPM organizations? Can this system support queries, such as those surrounding disparities in access to care? Can the system track changes longitudinally to allow for quality metrics reporting?

\section{Methods and Approach}

\section{Design}

This infrastructure development project began as a collaboration between a community-based HPM organization (Four Seasons, Flat Rock, NC) and an academic medical center (Duke University Medical Center, Durham, NC). Its ultimate purpose is to create a regional palliative care database that: (1) enables longitudinal tracking of outcomes, as well as analyses of patient characteristics; (2) supports quality assessment and monitoring; (3) flexibly meets the needs of diverse hospice and palliative care organizations; (4) helps these organizations respond to emerging reporting requirements; and (5) assists with workforce planning.

Stakeholders met to determine the approach to database development, data elements to be included, and individual site needs. Members from Four Seasons and Duke, as well as leaders from University of North Carolina-Chapel Hill (UNC), and local HPM organizations that would be potential contributors to/users of the database in the future, agreed on a modular approach which consisted of a set of core data elements with capacity to customize by adding site-specific data elements. Data items were intended to be meaningful across the continuum of HPM, so that the dataset could bridge inpatient and outpatient palliative care and hospice. Data elements were harmonized with those used at UNC, in order to preserve data interoperability for future research projects.

A data dictionary was developed; corresponding data collection forms were created and field-tested. A relational database was selected as the most practical solution. The architecture serves clinicians' needs, meets security requirements, and accepts data through a variety of data collection platforms including written data collection, tablet computers and digital pens. The database was initially programmed in Microsoft Access 2003 (Microsoft, Redmond, WA) for pilot testing purposes; it is being transferred to Microsoft SQL Server 2005 as confidence in the approach has progressed. Software, secure data transfer protocols, and data control procedures were developed. Data travel from the community site to the academic site for analysis and reporting; iterative feedback informs system refinement and directs analytic inquiries. A project wiki site provides bidirectional access to materials and facilitates communication.

\section{Setting}

Four Seasons is a nonprofit hospice and palliative care organization first established in 1978. A member of the National

TAble 1. Demographics

\begin{tabular}{|c|c|c|}
\hline & $\mathrm{n}$ & $\%$ \\
\hline \multicolumn{3}{|l|}{ Gender $(n=944)$} \\
\hline Female & 529 & 56 \\
\hline \multicolumn{3}{|l|}{ Race $(n=944)$} \\
\hline Caucasian & 868 & 92 \\
\hline African American & 33 & 3 \\
\hline Hispanic & 6 & 1 \\
\hline Native American & 2 & $<1 \%$ \\
\hline Asian/Pacific Islander & 1 & $<1 \%$ \\
\hline Other & 4 & $<1 \%$ \\
\hline Unknown & 30 & 3 \\
\hline \multicolumn{3}{|l|}{ Age at enrollment $(n=944)$} \\
\hline$>65$ years & 760 & 81 \\
\hline \multicolumn{3}{|l|}{$\begin{array}{l}\text { Prognosis at } \\
\quad \text { enrollment }(n=970)\end{array}$} \\
\hline$>6$ months & 437 & 45 \\
\hline \multicolumn{3}{|l|}{$\begin{array}{l}\text { Referring services } \\
\text { to hospice }(n=886)\end{array}$} \\
\hline Family practice & 253 & 29 \\
\hline Internal medicine & 228 & 26 \\
\hline Hospitalist & 171 & 20 \\
\hline Psychiatry/neurology & 88 & 10 \\
\hline Pulmonology & 60 & 7 \\
\hline Oncology & 24 & 3 \\
\hline Other & 62 & 5 \\
\hline \multicolumn{3}{|l|}{$\begin{array}{l}\text { Hospitalizations prior } \\
\text { to hospice enrollment }\end{array}$} \\
\hline No prior hospitalizations & 274 & 33 \\
\hline 1 prior hospitalization & 244 & 29 \\
\hline$\geq 2$ prior hospitalizations & 149 & 18 \\
\hline Unnknown & 175 & 20 \\
\hline \multicolumn{3}{|l|}{ Primary diagnoses } \\
\hline by ICD-9 codes $(n=822)$ & & \\
\hline Dementia & 214 & 26 \\
\hline $\begin{array}{l}\text { Chronic obstructive } \\
\text { pulmonary disease }\end{array}$ & 107 & 13 \\
\hline Congestive heart failure & 99 & 12 \\
\hline Cerebral vascular accident & 49 & 6 \\
\hline Lung cancer & 25 & 3 \\
\hline Altered mental status & 33 & 4 \\
\hline Breast cancer & 16 & 2 \\
\hline Aspiration pneumonia & 8 & 1 \\
\hline Other & 271 & 33 \\
\hline
\end{tabular}




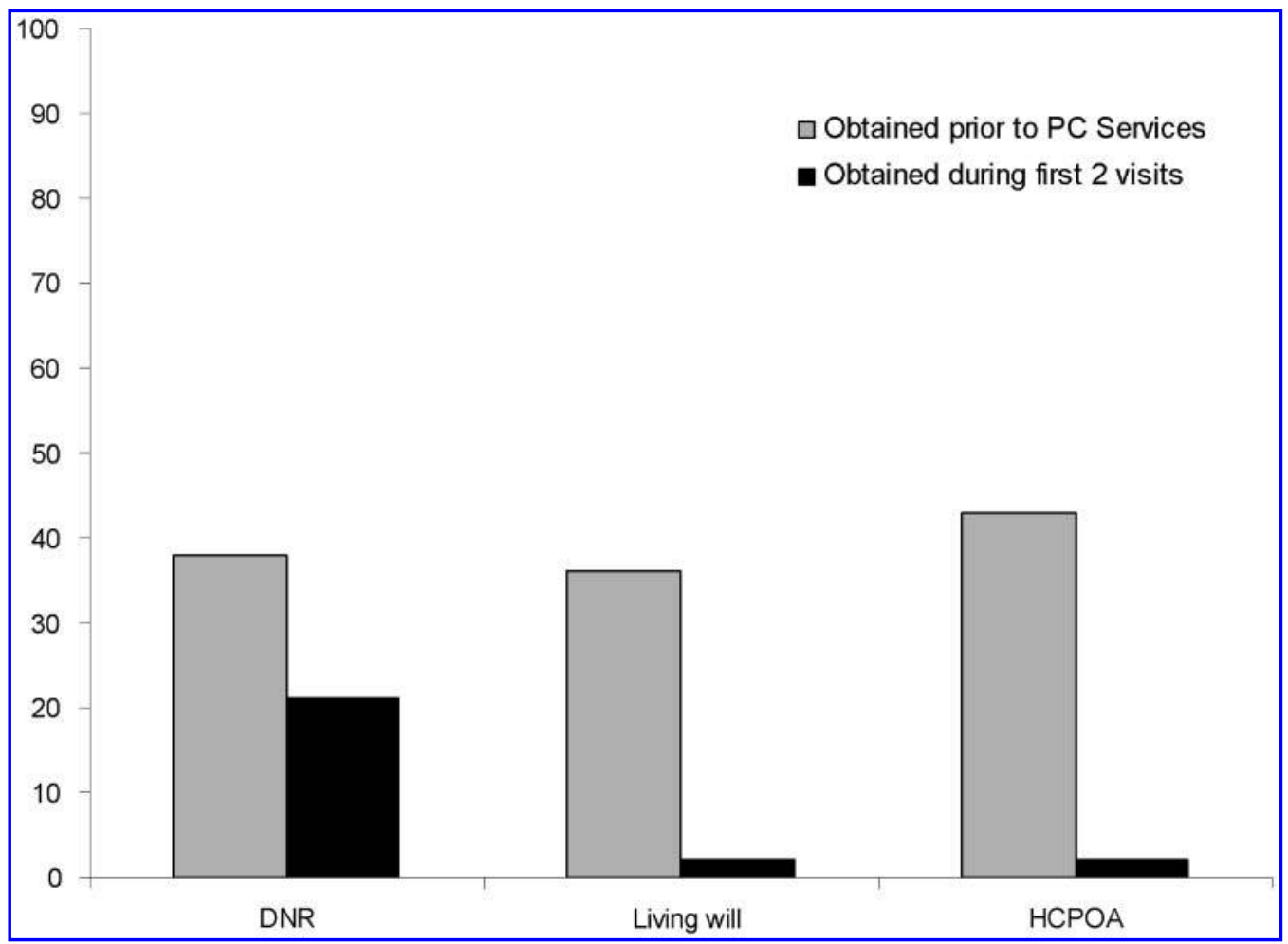

FIG. 1. Proportion of patients with advance care planning obtained prior to palliative care (PC) services or obtained during first 2 PC visits. $\mathrm{N}=970$. $\mathrm{DNR}=$ Do not resuscitate order; $\mathrm{HCPOA}=$ Health care power of attorney.

Hospice Work Group, Four Seasons received the 2009 Circle of Life Award. In 2008, Four Seasons served $72 \%$ of deaths in its surrounding area (Henderson County, NC) with hospice and palliative care services. As one of the early outpatient palliative care programs, it offers services to patients in the home, 21 nursing homes, 30 assisted living facilities, and its own 19-bed inpatient facility. Four Seasons has established an active research department that collaborates extensively with palliative care and oncology investigators at Duke.
Four Seasons was the first pilot site, and data are presented from that site. As this project evolved, three additional outpatient palliative care sites in North Carolina were recruited to pilot collaborative interactions. Reflecting the diversity in composition of palliative care programs, these sites represent outpatient, inpatient and blended HPM models of care; they are located in geographically dispersed and distant areas of North Carolina. Through the inclusion of additional sites, we plan to form a regional network of diverse HPM provider

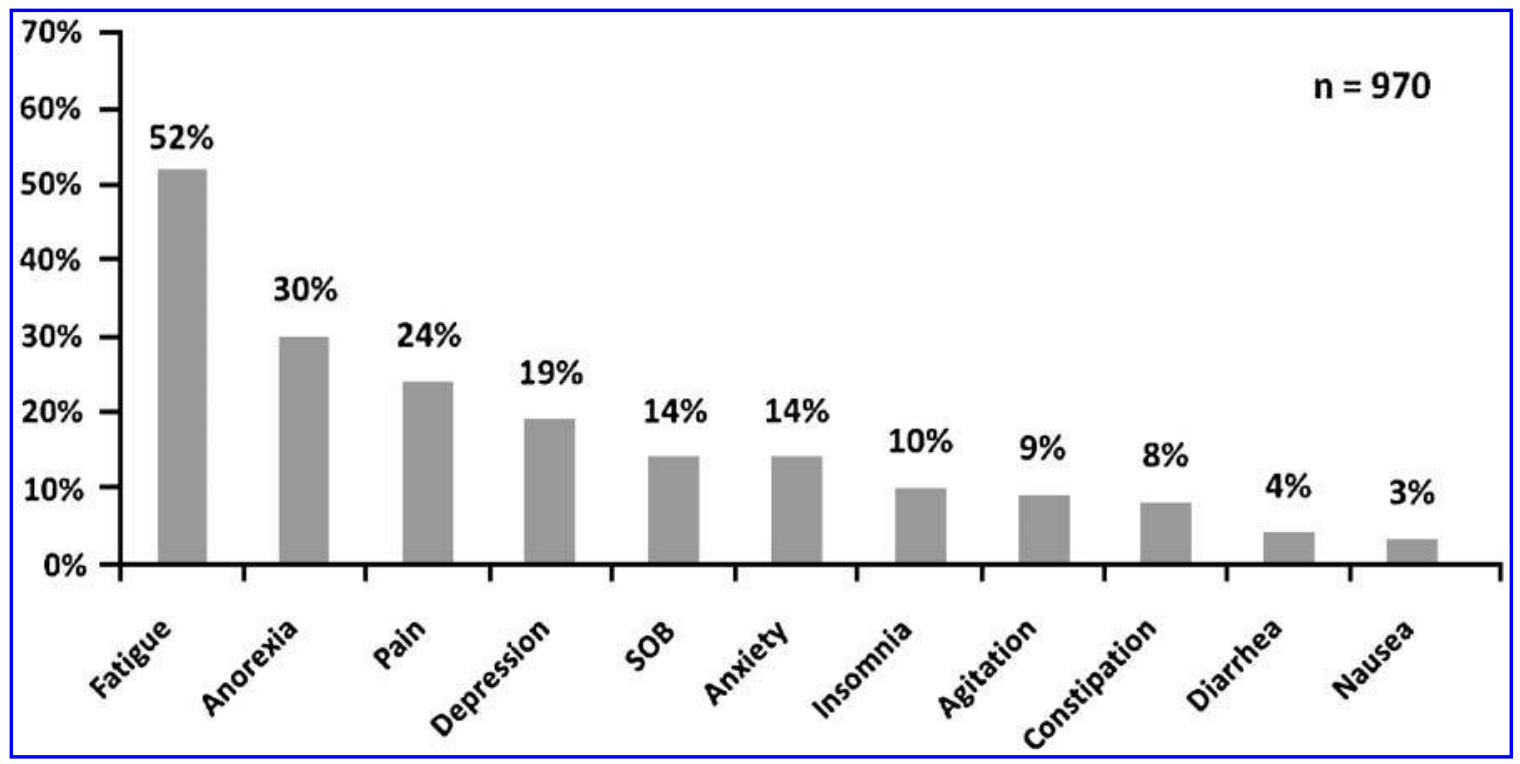

FIG. 2. Moderate to severe symptoms Includes patient, caregiver and clinician assessments. 
Table 2. Symptom Profiles from First AND SECOND Visits $(N=602)$

\begin{tabular}{lrr}
\hline & Visit 1 & Visit 2 \\
\hline Pain & & \\
Not a problem & $54 \%$ & $53 \%$ \\
Mild & $22 \%$ & $27 \%$ \\
Moderate-severe & $22 \%$ & $17 \%$ \\
Not assessed & $2 \%$ & $3 \%$ \\
Dyspnea & & \\
Not a problem & $66 \%$ & $68 \%$ \\
Mild & $18 \%$ & $18 \%$ \\
Moderate-severe & $12 \%$ & $8 \%$ \\
Not assessed & $4 \%$ & $6 \%$ \\
Depression & & \\
Not a problem & $57 \%$ & $58 \%$ \\
Mild & $18 \%$ & $21 \%$ \\
Moderate-severe & $19 \%$ & $12 \%$ \\
Not assessed & $6 \%$ & $9 \%$ \\
Constipation & & \\
Not a problem & $73 \%$ & $74 \%$ \\
Mild & $13 \%$ & $7 \%$ \\
Moderate-severe & $9 \%$ & $7 \%$ \\
Not assessed & $4 \%$ & \\
\hline
\end{tabular}

organizations across North and South Carolina-the Carolinas Consortium.

\section{Implementation}

Project implementation took place in two phases (Year 1, Year 2). Year 1 activities focused on development of the comprehensive data collection system. Four Seasons served as the pilot site. Key tasks were to: define data elements, develop a data dictionary, program the data elements for use with tablet personal computers (the initially selected technology platform for data collection), integrate the data collection software with the existing data management software at Four Seasons, practice point-of-care data collection, establish secure channels for data transfer from the Four Seasons server to the Duke server, complete programming for data management at the academic site, collect data on 200 new patients, and perform preliminary analyses on these data. Data are collected by mid-level or physician providers using paper case report forms, tablet computers, or electronic pens with corresponding data collection forms.

Year 2 goals were to: obtain commitment from three additional sites to join the project and contribute data; conduct

Table 3. Proportion of those Reporting Moderate/Severe Symptom Concern at Visit 1 with Reduction to Mild/None by Visit $2(N=602)$

\begin{tabular}{|c|c|c|c|}
\hline & $\begin{array}{l}\text { Patients with } \\
\text { moderate/severe } \\
\text { score at Visit } 1\end{array}$ & $\begin{array}{c}\text { Patients } \\
\text { with reduction } \\
\text { to mild/none } \\
\text { by Visit } 2\end{array}$ & $\begin{array}{c}\% \text { with } \\
\text { reduction in } \\
\text { symptom concern }\end{array}$ \\
\hline Pain & 133 & 75 & $56 \%$ \\
\hline Dyspnea & 75 & 49 & $65 \%$ \\
\hline Depression & 114 & 61 & $54 \%$ \\
\hline Constipation & 57 & 37 & $65 \%$ \\
\hline
\end{tabular}

an onsite training seminar; provide ongoing mentoring to the additional sites; develop a file transfer protocol for use at the additional sites; develop a model for collaboration and data governance, to guide data-sharing across sites; enter data on new patients; and develop quality initiatives based on evaluation of aggregate data.

\section{Current Status and Results \\ Data collection challenges}

This project set out to establish a new data collection system in a field not historically oriented toward research, or metrics more generally. ${ }^{2}$ The newness of data processes to HPM was apparent throughout implementation. Field testing of the data collection form resulted in four successive iterations before we arrived at a feasible and useful form. Difficulty with real-time access to data under the existing computerized data management system (Suncoast Solutions) at Four Seasons led to the development of a stand-alone database system using Microsoft Access. The mountainous terrain in western North Carolina caused wireless signal to be lost when collecting data via the planned technology platform (tablet computers); a docking system was instituted. Electronic pens are compact, inexpensive, and convenient, improve work flow, and function faster than the tablet computers, with quick download and validation time. They posed less of a barrier between patient and clinician, and achieved better clinician buy-in.

\section{Expanding into Carolinas Consortium}

In Year 1, eight palliative care providers at Four Seasons collected data using tablet computers and/or a paper version of the same data collection form. Data were stored on a local server and transferred monthly via a file transfer protocol to the server located at Duke. Between February and August 2008, providers entered data on 252 new patients. Initial data analyses, as proof of concept, were completed in September 2008; the analysis process was automated to facilitate future reporting. In Year 2, three additional sites began contributing data to the database: Horizons Palliative Care/Hospice of Wake County (Raleigh, NC), Hospice and Palliative Care Charlotte Region (Charlotte, NC), and Forsyth Medical Center Palliative Care Program (Winston-Salem, NC). Four Seasons hosted a 2-day training workshop in February 2009; 18 representatives from all four sites and Duke and UNC participated. In Year 2, Four Seasons added 970 additional patients into the database by June 2009; consequently, the system contained data from 2572 total visits.

\section{Symptom and demographic data}

Data from Four Seasons were tabulated to demonstrate how a regional data system could inform local understanding of palliative care patients and care received. Demographics of the first group of 970 patients are detailed in Table 1. We focused on individuals entered by Four Seasons into the system in Year 2, since most data quality concerns for this site were resolved in Year 1. Even so, the changing total numbers for each category presented in Table 1 reflect the challenge of missing data inherent with routine data collection embedded in clinical practice; iterative enhancements in technology and procedures reduced missing data over time. Prior to hospice 
enrollment, less than half of the patients had obtained a do-not-resuscitate (DNR) order, a living will, or a health care power of attorney (Fig. 1).

Symptom data were collected from the patient whenever possible. If the patient was unable to report, then caregiver or clinician assessment was documented, using a standardized approach with a flag to indicate who reported the information. Symptom profiles were explored (Fig. 2). Moderate to severe pain (rated $\geq 5$ on a $0-10$ scale) was reported by $22 \%$ of the 602 patients able to provide the information themselves on the first visit. Patient-reported rate is comparable to assessments of clinicians, caregivers and patients together, who identified moderate to severe pain in $24 \%$ of all patients $(n=970)$. Similar analyses were performed to determine the prevalence of other common symptoms (Table 2). Of those who reported moderate to severe pain, palliative care providers treated 55\% with pharmaceutical interventions, $7 \%$ with nonpharmaceutical interventions.

Data were collected during each patient's first and all subsequent visits. When the patient was in the hospital or being visited daily, data were collected for the first 3 days and then with clinical change, change in care location, or as indicated according to provider discretion. This approach allowed for comparison of symptom scores over time. Comparison of symptom scores at the first and second palliative care visits $(n=602)$ revealed that $56 \%$ of patients with moderate to severe pain at Visit 1 reported a reduction of pain severity to mild or none at Visit 2; similar reduction was reported for dyspnea (65\%), depression (54\%), and constipation $(65 \%)$ (Table 3).

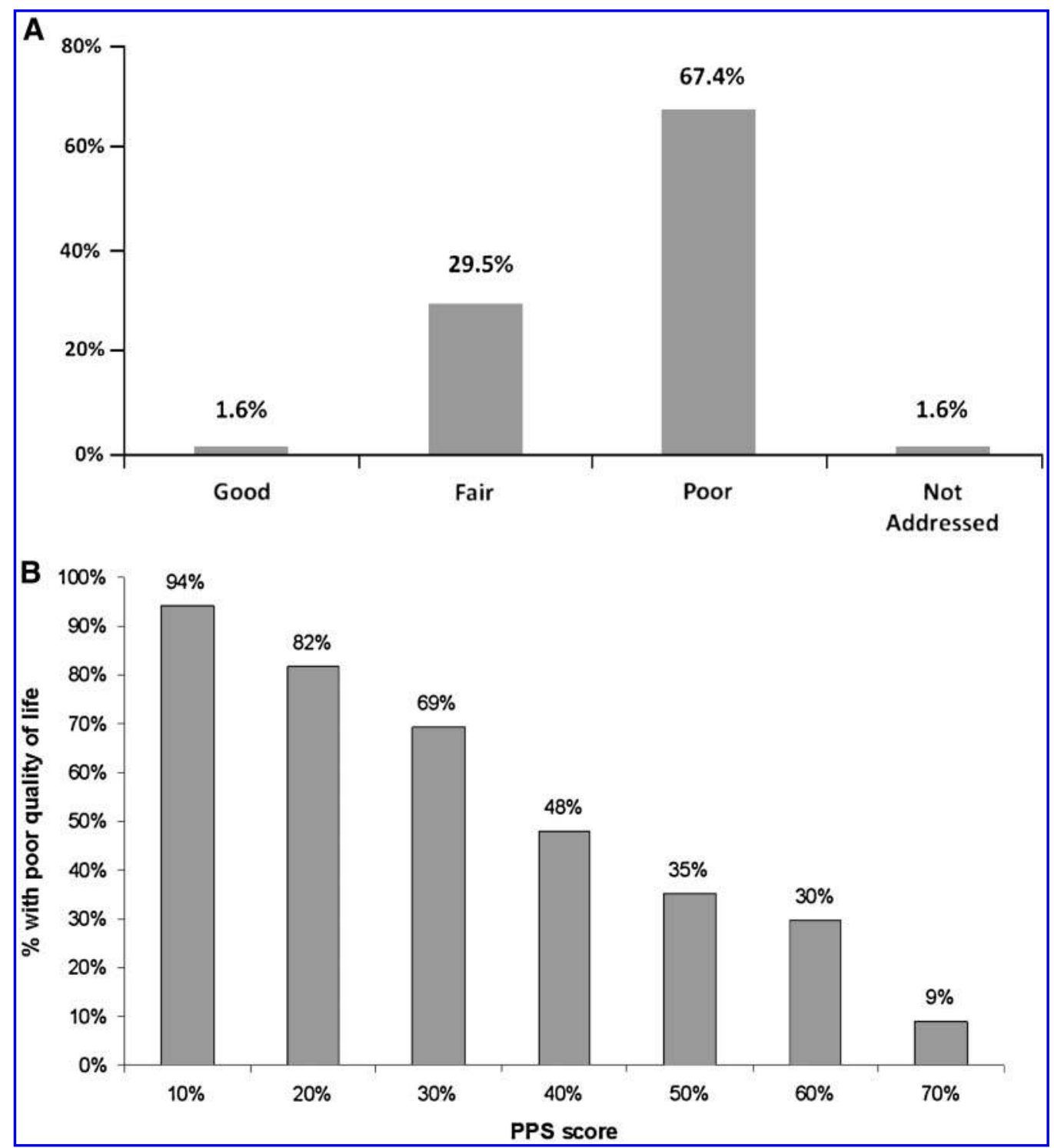

FIG. 3. (A) Reflects patients who have a prognosis of $\leq 6$ months (hospice eligible) Assessment by patient, caregiver and clinician. (B) Palliative Performance Status score and poor Quality of Life (QOL). QOL was subjectively scored by either caregiver or patient as poor, fair, good, or excellent. The percentages shown on the X-axis represent PPS percent score. The percentages shown in the bars represent percent of patients with the respective PPS percent score (e.g., 94\% of patients with a PPS of $10 \%$ have a poor QOL). 


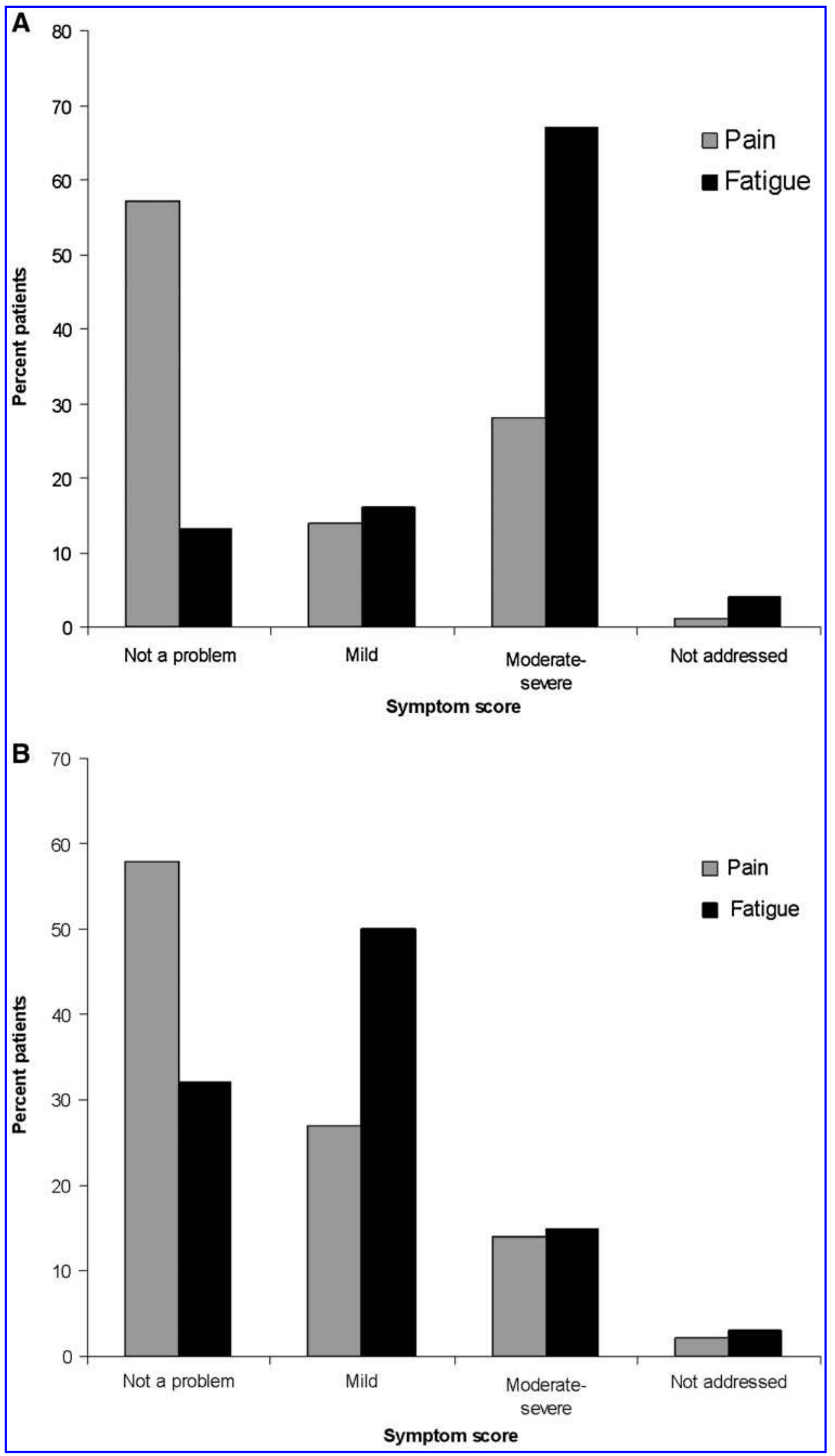

FIG. 4. (A) Proportion of patients with poor quality of life $(\mathrm{N}=425)$ who have pain and fatigue. (B) Proportion of patients with good quality of life $(\mathrm{N}=66)$ who have pain and fatigue. 


\section{Quality of life and functional status data}

The potential association between quality of life (QOL) and hospice eligibility, performance status, pain, and fatigue was evaluated. QOL was subjectively reported by the patient as poor, fair, good, or excellent. If the patient was unable to report, the caregiver or clinician assessed QOL. For patients with a clinician-assessed prognosis of less than 6 months $(n=438), 67 \%$ rated their QOL as poor (Fig. 3A). Functionality assessments were determined using the Palliative Performance Status (PPS) scale. There was a consistent relationship between PPS and QOL (Fig. 3B); mean PPS was 37\% for patients with poor QOL, 50\% for patients with fair QOL, and $60 \%$ for patients with good QOL. Among patients with poor QOL $(n=425), 28 \%$ had moderate to severe pain, and $67 \%$ had moderate to severe fatigue. In contrast, among the smaller group of patients who reported good QOL $(n=66), 14 \%$ had moderate to severe pain, $15 \%$ moderate to severe fatigue (Fig. $4 \mathrm{~A}$ and $4 \mathrm{~B})$.

\section{Disease-specific and race-/ethnicity-specific data}

The data were examined by diagnosis in order to identify quality improvement (QI) needs. Cardiac diagnoses (ICD-9 codes $401.9,414,427.31,428,428.42,428.9)$ pertained to $12 \%$ of the patient population; $20 \%$ of these patients reported moderate to severe pain, indicating a need for QI efforts in this area. For patients with chronic obstructive pulmonary disease (chronic obstructive pulmonary disease [COPD], ICD-9 code 496), representing $13 \%$ of the total population, $20 \%$ had moderate to severe pain and $25 \%$ indicated that they would desire a full code (versus DNR); both proportions prompted QI efforts at Four Seasons.

Three percent of consults were with African Americans, corresponding closely to the proportion of the population in the local service area who are African American (4\%). While most symptoms were reported at comparable rates across racial/ethnic groups, constipation appeared to be more of a problem for African Americans than for the total population (Fig. 5). The relatively small sample size of this pilot study precludes analysis; however, our initial exploration showed that disparities in symptom treatment and advance care planning can be identified.

\section{Discussion}

The development of outpatient palliative care delivery models adds new components to the continuum of hospice and palliative care services, allowing for more seamless service across care settings, supported transitioning from one setting to another, and inclusion of a greater proportion of patients with advanced illness who need supportive services. With the establishment of these new delivery models, it is now important to gain a clear understanding of (1) the population being served and (2) outcomes of care. These data will serve quality initiatives and administrative purposes, and will lay the groundwork for future research endeavors. Creation of a regional pooled data approach, as piloted here, represents a major first step in building this capacity.

This project yielded important lessons that will guide further efforts to build research and evaluation capacity in HPM. Organizational support and provider buy-in are imperative,

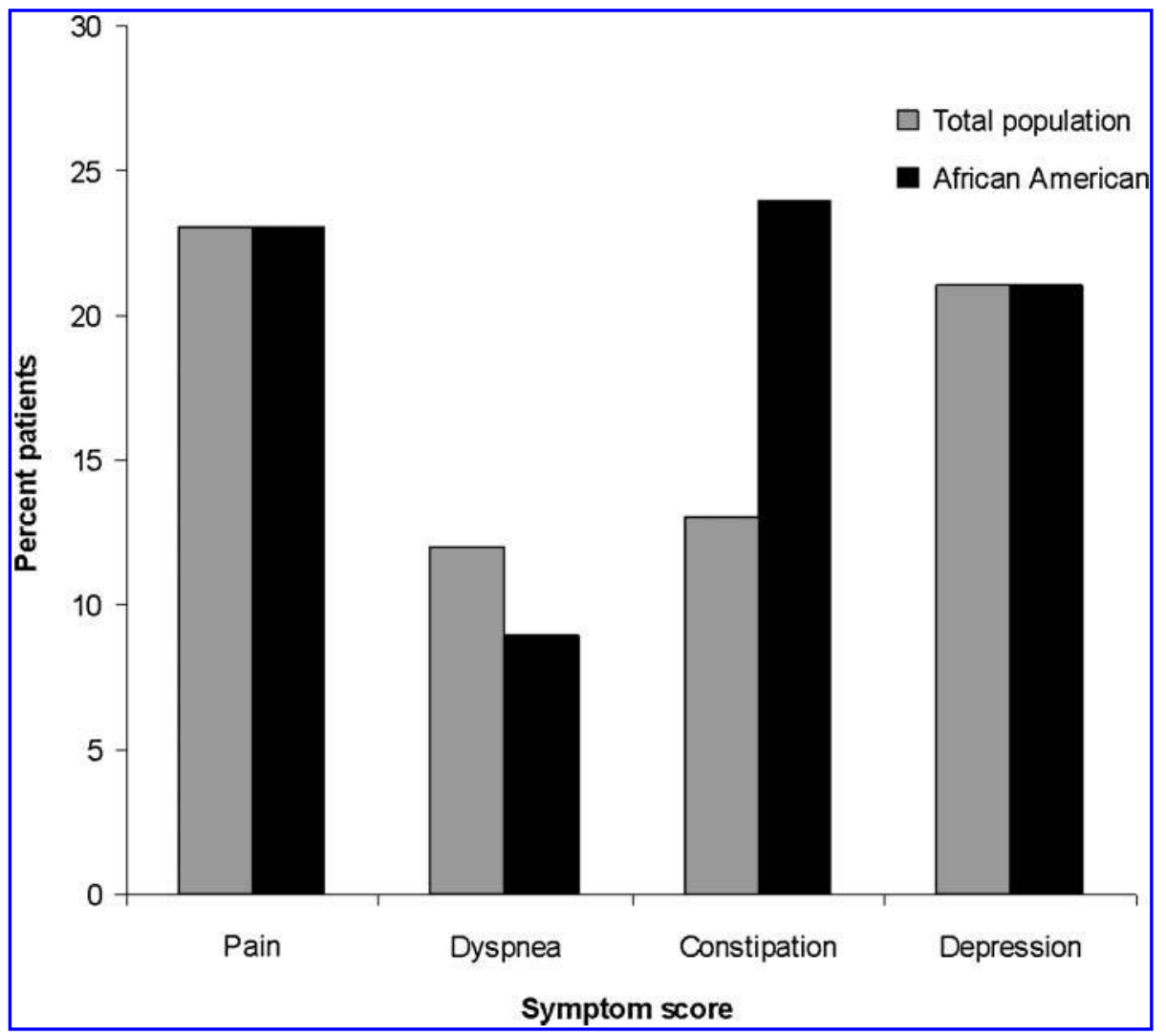

FIG. 5. Moderate to severe symptom prevalence in African American $(n=33)$ vs. total population $(n=970)$. 
as is the site-based users' input. Focus groups conducted with clinicians led to multiple iterations in the project, and ultimately a feasible approach for each site based on local needs and characteristics. Missing data initially presented a serious concern; required data entry fields (e.g., e-pen forms) eliminated missing data. It is important to offer multiple data collection methods to sites since technology expertise and equipment vary from site to site. Equally important are technicians to set up the file transfer protocol and statisticians to help interpret data results. These success factors, identified in the course of implementing this pilot phase of the database, will guide its further development and streamline the addition and upskilling of new sites.

The data system proved useful for characterizing the population being served, yielding insights into disease and symptom burden, quality of life, distribution of end-of-life planning, and other metrics related to these patients. Hypothesis-generating questions such as the association between QOL and symptoms were explored, demonstrating the potential research utility of the system. As the database grows to include a large number of patients over a sizeable geographic area, this utility will increase. We will be able to compare data in aggregate, across sites, by provider, or for individual patient types. Creation of a regional pooled data approach, as piloted here, represents a major first step in building this capacity.

Preliminary analyses of the data generated immediate practical information. Examination of demographics revealed that, while Henderson County, NC, comprises 11\% Hispanic individuals, the Four Seasons new patient population included only $1 \%$ Hispanic patients-indicating a need for outreach and/or QI efforts to better reach this segment of the population. Additionally, 55\% of patients reporting moderate to severe pain were treated with pharmacological intervention prescribed by Four Seasons; $7 \%$ of these patients received treatment through nonpharmacologic measures. This demonstrates the impact palliative care has on pain management.

A vital outcome of this project was creation of the Carolinas Consortium, now including the four sites contributing to the database, and intended to include additional sites as they join the data collection activities. To cultivate this Consortium, member join biweekly conference calls for participants share best practices and provide support in working out the details of implementing this data collection system in their organizations. Teleconferences have established a working-group approach that facilitates tasks such as defining quality metrics and iteratively improving the database processes.

This project has several limitations. The newly created database contains a limited, though growing, number of patients and data points; its ability to describe this population is correspondingly limited, although improving. Missing data might not be at random, potentially leading to systematic exclusion of important patient populations (e.g., poor cognition or performance status). We found the greatest proportion of missing data around acceptable and worst level of pain and shortness of breath, and number of previous hospitalizations; reasons for this pattern will be explored. Validation studies to understand and improve data quality are planned. As more data are transferred to the warehouse and processes formalized, our understanding of data quality will improve and results will become progressively more meaningful. Such endeavors will help HPM programs prepare for national quality initiatives such as the Centers for Medicare and Medicaid Services Physician Quality Reporting Initiative; they might ultimately contribute to research into best practice for HPM.

These findings are preliminary. The intention of this article is to demonstrate that data can be collected in diverse HPM settings, and that the data can yield useful information. Standardization of data collection has been a focus of training, but has likely been imperfect in the course of this study. Future efforts will focus on creating feasible methods that ensure a standardized data collection approach. Challenges to the implementation of the project's data collection methods included clinician buy-in and commitment and financial impact of adding the related tasks. Many HPM organizations lack budgetary flexibility to purchase new technologies for data collection or cover personnel time required for data entry; motivation to collect data may remain low until organizations face a requirement to report on quality metrics. Finally, a sustainability plan is needed to support database operation, provide technical support, perform data analysis, and support reporting.

Despite these practical hurdles, the Carolinas Consortium is a successful collaborative regional model for data collection and interpretation to improve the quality of care that we provide in the growing palliative care environment. We anticipate advancements at the site, Consortium, and state levels, including better work force planning. Community and academic partnerships strengthen our ability to analyze results and identify next steps for improvement. Communication among sites to inform database development has had corollary impacts including sharing best practices and support for evidence-based medicine.

\section{Acknowledgments}

Funding for this project was generously provided by a philanthropic grant from The Duke Endowment.

\section{Author Disclosure Statement}

No competing financial interests exist.

\section{References}

1. Meier DE, Beresford L: Outpatient clinics are a new frontier for palliative care. J Palliat Med 2008;11:823-828.

2. Hanson LC, Schenck AP, Rokoske FS, et al. Hospice organizations' capacity for quality measurement. J Pain Symptom Manage (in press).

3. Dudgeon DJ, Knott C, Chapman C, Coulson K, Jeffery E, Preston S, Eichholz M, Van Dijk JP, Smith A. et al. Development, implementation, and process evaluation of a regional palliative care quality improvement project. J Pain Symptom Manage 2009;38:483-495.

4. Meier DE, Beresford L: Dartmouth Atlas data can support palliative care development. J Palliat Med 2008;11:960-963.

Address correspondence to: Amy P. Abernethy, M.D. Duke University Medical Center Box 3436

Durham, NC 27710

E-mail: amy.abernethy@duke.edu 